\title{
Final Report on DOE Grant DE-SC0001075 titled "Supersymmetry and Unification of Fundamental Interactions-SUSY2009
}

Final report for period: 06/2009- 05/2010

Principal Investigator: Nath, Pran

Organization: Northeastern University

Submitted By: Nath, Pran - Principal Investigator

Title: Supersymmetry and Unification of Fundamental Interactions-SUSY2009

\section{Project participants}

\section{Senior Personnel}

Name: Nath, Pran

Other Participants: Nearly 350 conference participants including senior faculty, junior faculty, post docs, and graduate students.

\section{Activities and Findings}

SUSY2009 brought together researchers from a very broad area of fundamental physics including elementary particle theory, astrophysics and cosmology and string theory with a focus on supersymmetry. Thus the topic covered at the SUSY2009 conference included the following:

Search for the Higgs boson, search for supersymmetry, supersymmetry phenomenology, theories of dark matter and direct and indirect detection, neutrino physics, accelerator experiments, electroweak physics, supersymmetry phenomenology, string theory, string phenomenology, extra Dimensions as well as other recent theoretical and experimental developments. The conference was successful in fostering interdisciplinary interactions between theorists and experimentalists.

\section{Training and Develpment}

The project provided training for many graduate student who participated in the conference to learn how science is done by listening to leading experts in several front line areas of particle physics. 


\section{Outreach Activities}

A public lecture by Nobel Laureate Frank Wilczek was arranged which was geared to a broad audience to make the public aware of the progress occurring in the field of fundamental science in this area.

\section{Publications}

"Supersymmetry and the unification of fundamental interactions. Proceedings, 17th International Conference, SUSY09, Boston, USA, June 5-10, 2009," edited by P. Nath, (ed.), G. Alverson, (ed.) and B. Nelson, (ed.), C09-06-05 (AIP Conf. Proc. 1200 (2009) 1159 p). The AIP publication includes the latest developments on the topics discussed above which include the Higgs boson, search for supersymmetry, supersymmetry phenomenology, theories of dark matter and direct and indirect detection, neutrino physics, accelerator experiments, electroweak physics, supersymmetry phenomenology, string theory, string phenomenology, and extra Dimensions. Both theoretical and experimental talks were included. 


\title{
Conference Agenda
}

\author{
$+$ \\ List of Attendees
}




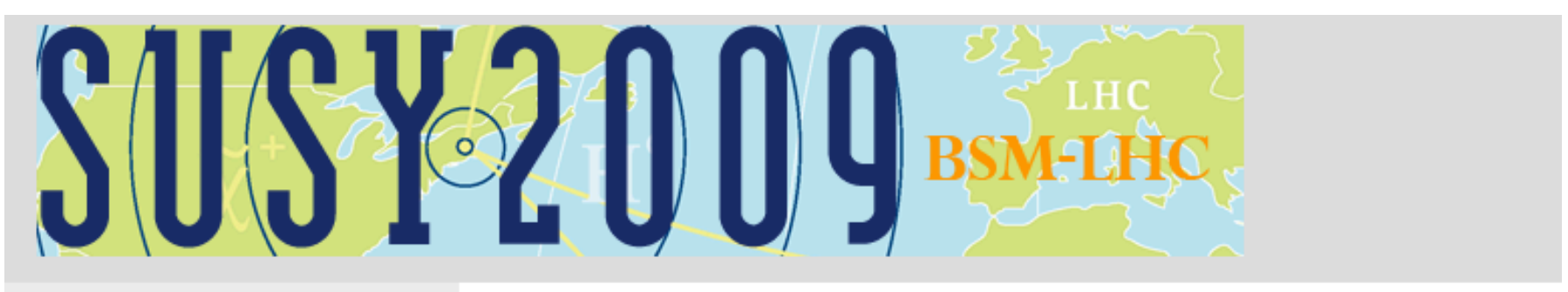

SUSY09: 5-10 June BSM-LHC: 2-4 June

Home

Registration

Abstract Submittal

Off-campus Lodging

Proceedings

Titles/Abstracts

Attendees

Session Chairs

\section{SUSY '09 Plenary Schedule}

\begin{tabular}{|c|c|}
\hline \multicolumn{2}{|r|}{ Friday } \\
\hline & Session 1 (Friday June $5,8: 30$ AM) \\
\hline \multicolumn{2}{|r|}{ Chair: Pran Nath } \\
\hline \multicolumn{2}{|l|}{ Registration } \\
\hline \multicolumn{2}{|l|}{ Welcome } \\
\hline Jenni & LHC Entering Operation: an Overview of the LHC Program \\
\hline D'Onofrio & Experimental searches for SUSY at the Tevatron \\
\hline
\end{tabular}

Coffee Break (Friday June 5, 10:45 AM)

Session 2 (Friday June 5, 11:15 AM)

Chair: George Alverson

Büscher Experimental Searches for Higgs Bosons at the Tevatron

Baer Leptonic SUSY signatures at the LHC

Lunch (Friday June 5, 12:45 PM)

Session 3 (Friday June 5, 2:15 PM)

Chair: Haim Goldberg

Dreiner

How light can the lightest Neutralino be?

Antoniadis

Anomaly driven physics of extra U(1)'s

Sundrum

Coffee Break (Friday June 5, 3:45 PM)

Session 4 (Friday June 5, 4:10 PM)

Chair: Michael Vaughn

Cvetic D-Instantons and Particle Physics Implications

Raby

String Model Building

de Boer Indirect Dark Matter Signatures in the light of FERMI, EGRET and PAMELA

\begin{tabular}{cc}
\hline & Reception (Friday June 5, 6:30 PM) \\
\hline & Monday \\
\hline & Session 5 (Monday June 8, 9:00 AM) \\
Chair: Howard Haber \\
David & CMS: from commissioning to first beams \\
Jinnouchi & Searches for SUSY with the ATLAS detector \\
Mohapatra & Neutrino Mass Physics and Origin of Matter at LHC \\
\hline
\end{tabular}

Coffee Break (Monday June 8, 10:30 AM)

Session 6 (Monday June 8, 11:00 AM)

Chair: Pran Nath

Gaillard T-duality and the weakly coupled heterotic string

Randall Flavor Physics from Extra Dimensions

Nilles From the heterotic string to the MSSM

Lunch (Monday June 8, 12:30 PM) 
Session 7 (Monday June 8, 2:00 PM)

Chair: Michael Vaughn

$\begin{array}{cr}\text { Profumo } & \text { Fundamental Physics from the Sky } \\ \text { Pilaftsis } & C P \text { Violation in Supersymmetry } \\ \text { Tata } & \text { Supersymmetry, Dark Matter and the LHC }\end{array}$

Coffee Break (Monday June 8, 3:30 PM)

\begin{tabular}{|c|c|}
\hline \multicolumn{2}{|r|}{ Session 8 (Monday June 8, 4:00 PM) } \\
\hline \multicolumn{2}{|r|}{ Chair: Tomasz Taylor } \\
\hline Zumino & $\begin{array}{l}\text { Star Product for exterior differential forms with application } \\
\text { to superstring theory }\end{array}$ \\
\hline Dixon & Multi-Loop Finiteness of $N=8$ Supergravity \\
\hline \multicolumn{2}{|r|}{ Banquet (Monday June 8, 6:30 PM) } \\
\hline \multicolumn{2}{|r|}{ Tuesday } \\
\hline \multicolumn{2}{|r|}{ Session 9 (Tuesday June $9,8: 30$ AM) } \\
\hline \multicolumn{2}{|r|}{ Chair: Apostolos Pilaftsis } \\
\hline Klein & $\begin{array}{l}\text { Neutrinos from Fermilab, the Sky, the Earth, the Sun and } \\
\text { the Stars, to Homestake }\end{array}$ \\
\hline de Sangro & New Physics Searches at the B-Factories \\
\hline Kinoshita & $\begin{array}{l}\text { New Physics Searches at the B-Factories II: Examining the } \\
\text { Loops }\end{array}$ \\
\hline Masiero & CPV and Flavor in SUSY: Frustration and Hope \\
\hline
\end{tabular}

Coffee Break (Tuesday June 9, 10:30 AM)

\begin{tabular}{|c|c|}
\hline \multicolumn{2}{|r|}{ Session 10 (Tuesday June 9, 11:00 AM) } \\
\hline \multicolumn{2}{|r|}{ Chair: Herbi Dreiner } \\
\hline Zerwas & Extracting the fundamental parameters \\
\hline Langacker & The Physics of New $U(1)^{\prime}$ Gauge Bosons \\
\hline Babu & Recent Progress in SUSY GUTS \\
\hline \multicolumn{2}{|r|}{ Lunch (Tuesday June 9, 12:30 PM) } \\
\hline \multicolumn{2}{|r|}{ Session 11 (Tuesday June 9, 2:00 PM) } \\
\hline & Chair: Brent Nelson \\
\hline Kane & $\begin{array}{c}\text { Non-thermal wino LSP dark matter describes Pamela data } \\
\text { welll }\end{array}$ \\
\hline Wagner & The Supersymmetric Origin of Matter \\
\hline Buchmuller & Gravitino Dark Matter \\
\hline
\end{tabular}

Coffee Break (Tuesday June 9, 3:30 PM)

\begin{tabular}{|c|c|}
\hline \multicolumn{2}{|r|}{ Session 12 (Tuesday June 9, 4:00 PM) } \\
\hline \multicolumn{2}{|r|}{ Chair: Dieter Lüst } \\
\hline Shifman & $\begin{array}{c}\text { Non-Abelian Strings: From Weak to Strong Coupling and } \\
\text { Back via Duality }\end{array}$ \\
\hline Trivedi & $\begin{array}{c}\text { Non-Supersymmetric Stable Throats in Flux } \\
\text { Compactifications }\end{array}$ \\
\hline Valle & Neutrino mass in supersymmetry: Theory and implications \\
\hline \multicolumn{2}{|r|}{ Wednesday } \\
\hline \multicolumn{2}{|r|}{ Session 13 (Wednesday June 10, 9:00 AM) } \\
\hline \multicolumn{2}{|r|}{ Chair: Rabindra Mohapatra } \\
\hline Nandi & Fermion Mass Hierarchy and New Physics at the TeV Scale \\
\hline King & Neutrino Mass and Flavour Models \\
\hline Kim & Current status of axion physics \\
\hline
\end{tabular}

Coffee Break (Wednesday June 10, 10:30 AM) 


\section{Session 15 (Wednesday June 10, 2:00 PM)}

Chair: Hans Peter Nilles

\begin{tabular}{|c|c|}
\hline Senjanovic & Proton Decay and Grand Unification \\
\hline Dienes & $\begin{array}{c}\text { New Non-Trivial Vacuum Structures in Supersymmetric Field } \\
\text { Theories }\end{array}$ \\
\hline
\end{tabular}

†Professor Vafa's non-appearance was unintentional and resulted from a miscommunication with the Organizing Committee 


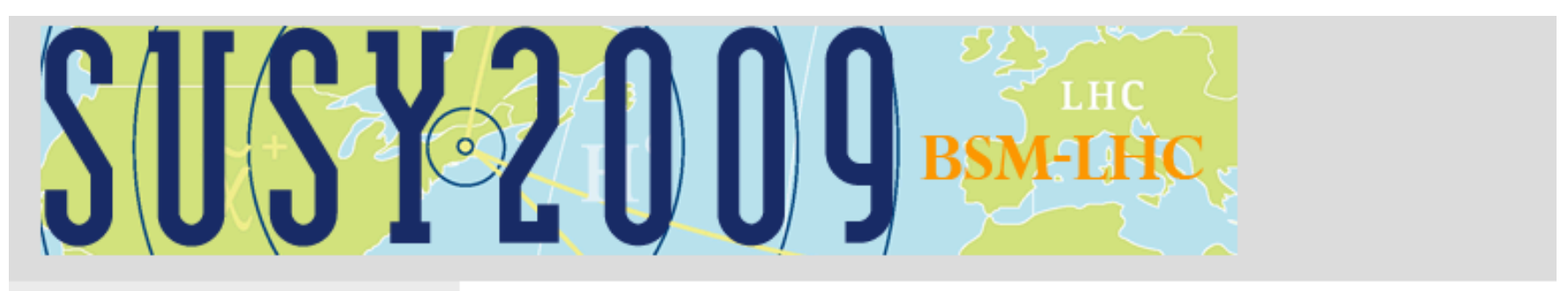

SUSY09: 5-10 June BSM-LHC: 2-4 June

Registration Abstract Submittal On-campus Lodging Off-campus Lodging Proceedings Attendees

\section{A List of SUSY09/BSM-LHC Attendees}

\begin{tabular}{|c|c|}
\hline Amir D. Aczel & Boston University \\
\hline $\begin{array}{l}\text { Claire } \\
\text { Adam-Bourdarios }\end{array}$ & LAL \\
\hline Elif Asli Albayrak & University of Iowa \\
\hline Martin Aleksa & CERN \\
\hline Tavanfar Alireza & Harvard \\
\hline Rouzbeh Allahverdi & University of New Mexico \\
\hline $\begin{array}{l}\text { Wolfgang } \\
\text { Altmannshofer }\end{array}$ & Technische Universitaet Muenchen \\
\hline Baris Altunkaynak & Northeastern University \\
\hline George Alverson & Northeastern University \\
\hline Johan Alwall & SLAC \\
\hline Luis Anchordoqui & UWM \\
\hline Alberto Annovi & INFN - Laboratori Nazionali di Frascati \\
\hline Ignatios Antoniadis & CERN - TH \\
\hline Eric Armengaud & CEA - IRFU \\
\hline Olivier Arnaez & $\begin{array}{l}\text { Laboratoire d'Annecy-le-Vieux de Physique } \\
\text { des Particules (LAPP) }\end{array}$ \\
\hline Peter Athron & Technische Universitat Dresden \\
\hline Kaladi Babu & Oklahoma State University \\
\hline Howard Baer & University of Oklahoma \\
\hline Yang Bai & Fermilab \\
\hline Travis Bain & University of Toronto/ATLAS \\
\hline Oliver Baker & Yale University \\
\hline Csaba Balazs & Monash University \\
\hline $\begin{array}{l}\text { Priyotosh } \\
\text { Bandyopadhyay }\end{array}$ & Harish-Chandra Research Institute \\
\hline Swagato Banerjee & University Of Victoria, Canada \\
\hline Anne-Fleur Barfuss & CPPM \\
\hline Nans Baro & Institut fuer Theoretische Physik E \\
\hline Alan Barr & University of Oxford \\
\hline Stephen Barr & University of Delaware \\
\hline Anders Basbøll & University of Sussex \\
\hline Rachid Benbrik & NCTS \\
\hline Catrin Bernius & University College London (UCL) \\
\hline Tracey Berry & Royal Holloway, University of London \\
\hline Marc Besancon & CEA-Saclay Irfu/SPP \\
\hline Aatish Bhatia & Rutgers University \\
\hline Anwar Bhatti & Rockefeller University \\
\hline Pietro Biassoni & Universita' degli Studi and INFN Milano \\
\hline Simone Bifani & University of Bern ITP \\
\hline Catherine Biscarat & CC-IN2P3 \\
\hline Steve Blanchet & University of Maryland \\
\hline Andrew Blechman & University of Toronto \\
\hline Freya Blekman & Cornell \\
\hline Nicki Bornhauser & University of Bonn \\
\hline Sascha Bornhauser & University of New Mexico \\
\hline Francesca Borzumati & National Taiwan University \\
\hline Andrew Box & University of Oklahoma \\
\hline Felix Braam & Freiburg University \\
\hline
\end{tabular}




\section{Xuebing $\mathrm{Bu}$}

Wilfried Buchmuller

Matthew Buican

Volker Büscher

Marco Cardaci

Marcela Carena

Linda Carpenter

Elizabeth Castaneda-

Miranda

Pierluigi Catastini

Richard Cavanaugh

David G. Cerdeno

Riccardo Cerulli

Zackaria Chacko

Hamad Chaudhry

Jie Chen

Mu-Chun Chen

Ning Chen

Kiwoon Choi

Ilias Cholis

Eung Jin Chun

Daniel Chung

Diego Julio Cirilo

Lombardo

Louis Clavelli

John Conley

Cristobal Cuenca

Almenar

Javier Cuevas

Yanou Cui

Ray Culbertson

Mirjam Cvetic

Mariarosaria D'Alfonso

Monica D'Onofrio

De-Chang Dai

Asesh K Datta

Andre David

Hooman Davoudiasl

Senarath de Alwis

Wim de Boer

Paul de Jong

Ivo de Medeiros

Varzielas

Guglielmo De Nardo

Riccardo de Sangro

Andrea De Simone

Pierre-Philippe

Dechant

Frank Deppisch

Cristinel Diaconu

Lorenzo Diaz-Cruz

Keith Dienes

Janet Dietrich

John Dixon

Lance Dixon

Alexandru Florin

Dobrin

Mikhail Dolgopolov
University of Science and Technology of China

DESY

Princeton University

Mainz University

Universiteit Antwerp

FERMILAB/ U. Chicago

UC Santa Cruz

University of Wisconsin-Madison

INFN Pisa / Siena University

Fermilab / University of Illinois at Chicago

Universidad Autonoma de Madrid

INFN-LNGS

University of Maryland, College Park

KTH

Florida State University

UC Irvine

Stony Brook

KAIST

New York University

Korea Institute for Advanced Study

University of Wisconsin - Madison

BLTP (JINR-Dubna/Russia)

University of Alabama

SLAC

UC Irvine

University of Oviedo

Harvard University

Fermilab

University of Pennsylvania

UCSB

Institut de Fisica d'Altes Energies (IFAE) -

Barcelona

SUNY at Buffalo

HRI, Allahabad

LIP, Lisbon

Brookhaven National Laboratory

University of Colorado

IEKP

Nikhef

CFTP, IST

Univ. of Napoli and INFN

INFN - LNF

MIT

Cambridge University

University of Manchester

CPPM/DESY

FCFM-BUAP

University of Arizona

Physikalisches Institut, University of

Freiburg

Dixon Law Firm

SLAC

Lund University

Samara State University, Dept. of Gen. and Theor. Physics 


\begin{tabular}{|c|c|}
\hline Aaron Dominguez & University of Nebraska -- Lincoln \\
\hline Mauro Donega & University of Pennsylvania \\
\hline Ilja Dorsner & Institute Jozef Stefan \\
\hline Herbi Dreiner & Bonn University \\
\hline Bhaskar Dutta & Texas A\&M University \\
\hline Hajar Ebrahim & Brandeis University \\
\hline Ulrich Ellwanger & Lab. de Physique Theorique \\
\hline Carlos Escobar & UNICAMP \\
\hline Rouven Essig & SLAC \\
\hline Adam Everett & Purdue University \\
\hline Lisa Everett & University of Wisconsin, Madison \\
\hline Marcello Fanti & Universita` degli Studi di Milano and INFN \\
\hline Daniel Feldman & Northeastern University \\
\hline WanZhe FENG & Northeastern Univ \\
\hline Alexey Ferapontov & Brown University \\
\hline $\begin{array}{l}\text { Enectali Figueroa- } \\
\text { Feliciano }\end{array}$ & Massachusetts Institute of Technology \\
\hline Pavel Fileviez Perez & University of Wisconsin-Madison \\
\hline Simon Fiorucci & Brown University \\
\hline Thomas Flacke & University of Michigan \\
\hline Henning Flaecher & CERN \\
\hline $\begin{array}{l}\text { Luis Roberto Flores } \\
\text { Castillo }\end{array}$ & University of Wisconsin \\
\hline Alison Fowler & Institute of Particle Physics Phenomenology \\
\hline Paul Frampton & University of North Carolina \\
\hline Sebastian Frank & $\begin{array}{l}\text { Institute of High Energy Physics (HEPHY), } \\
\text { Vienna }\end{array}$ \\
\hline Ayres Freitas & University of Pittsburgh \\
\hline Sky French & University of Cambridge \\
\hline James Frost & University of Cambridge \\
\hline Mary K Gaillard & UC Berkeley/LBNL \\
\hline James Gainer & SLAC \\
\hline Diego Gallego & SISSA/ISAS \\
\hline Jason Gallicchio & Harvard \\
\hline Robert Garisto & PRL \& BNL \\
\hline Cecilia Garraffo & $\begin{array}{l}\text { IAFE (Instituto de Astronomia y Fisica del } \\
\text { Espacio) }\end{array}$ \\
\hline Jan Germer & MPI für Physik München \\
\hline Marco Gersabeck & University of Glasgow \\
\hline Yuri Gershtein & Rutgers \\
\hline Tony Gherghetta & University of Melbourne \\
\hline Joel Giedt & RPI \\
\hline Elena Ginina & HEPHY, Vienna \\
\hline Franco Giuliani & University of New Mexico \\
\hline Vladimir Gligorov & University of Glasgow \\
\hline Ilia Gogoladze & University of Delaware \\
\hline Michael Gold & University of New Mexico \\
\hline Haim Goldberg & Northeastern University \\
\hline Mario E Gomez & Universida de Huelva \\
\hline Melina Gomez & Instituo de Fisica, UNAM \\
\hline Max Goncharov & MIT \\
\hline Shrihari Gopalakrishna & Brookhaven National Lab \\
\hline Stefania Gori & Technische Universitaet Muenchen \\
\hline Sebastian Grab & Bonn University \\
\hline Michael Graesser & LANL \\
\hline Peter Graham & Stanford \\
\hline Eilam Gross & Weizmann \\
\hline Yuval Grossman & Cornell \\
\hline John Gunion & U.C. Davis \\
\hline Sudhir Gupta & Iowa State University \\
\hline
\end{tabular}


Howard Haber

Haleh Hadavand

Tao Han

Zhenyu Han

Koji Hara

Sam Harper

Helen Hayward

Sven Heinemeyer

Johannes Heinonen

Jaime Hernández-

Sánchez

Maria Herrero

Keisho Hidaka

Michael Holmes

Tuomas Honkavaara

Claus Horn

Sabine Hossenfelder

Richard Howl

Raghavendra Hundi

Hyolung Hyun

John Idarraga

Ahmad Idilbi

Kyoko Iizuka

Amon Ilakovac

Antonella Incicchitti

Tadaaki Isobe

Cigdem Issever

Andrew Ivanov

Shabnam Jabeen

Peter Jenni

Osamu Jinnouchi

Chad Johnson

Dong-Won Jung

Dilani Kahawala

Teruki Kamon

Gordon Kane

Georgia Karapostoli

Alex Kastanas

Yevgeny Kats

Panagiotis

Katsaroumpas

Andrey Katz

Gregory Keefer

Joern Kersten

Clemens KieBig

Tatsuru Kikuchi

Jennifer Kile

Chul Kim

Hyung Do Kim

Ian-Woo Kim

Jihn E. Kim

Yeong Gyun Kim

Steve King

Kay Kinoshita

Joshua Klein

Ameya Kolarkar

Yury Kolomensky

Tadashi Kon

Otto Kong
University of California, Santa Cruz

Southern Methodist University

Univ. of Wisconsin

UC Davis

Nagoya University

Rutherford Appleton Laboratory (STFC), UK

University of Liverpool

IFCA (CSIC)

Cornell University

FCE-BUAP

Universidad Autonoma de Madrid

Tokyo Gakugei University

Northeastern

Helsinki Institute of Physics

SLAC

Perimeter Institute

University of Southampton

University of Hawaii

Kyungpook National University

York University

Duke University

Seikei Univ.

University of Zagreb, Faculty of Science

INFN sezione di Roma

University of Tokyo

University of Oxford

UC Davis

Boston University

CERN

KEK

Columbia University

National Central University

Harvard University

Texas A\&M University

University of Michigan

Imperial College London

University of Bergen

Harvard University

Queen Mary, University of London

University of Maryland

The University of Alabama

University of Hamburg

MPI of physics, Munich

KEK

BNL

Duke University

Seoul National University

University of Wisconsin-Madison

Seoul National University

KAIST

University of Southampton

University of Cincinnati

University of Pennsylvania

Boston University

LBNL

Seikei Univ.

National Central University 
Daniel Koschade

Philipp Kostka

Dmytro Kovalskyi

Karol Kovarik

James Kraus

Graham Kribs

David Krohn

Jason Kumar

Piyush Kumar

Masafumi Kurachi

Florent Lacroix

Caleb Lampen

Greg Landsberg

Paul Langacker

Ulrich Langenfeld

David Larson

Tomas Lastovicka

Alfio Lazzaro

Cristina Lazzeroni

Fabienne Ledroit-

Guillon

Hye-Sung Lee

Federica Legger

Liang Li

Syue-Wei Li

Yingchuan Li

Roman Linares

Mariangela Lisanti

Tao Liu

Zuowei Liu

Magda Lola

Owen Long

David Lopes Pegna

David Lopez Mateos

Sotiris Loucatos

Ian Low

Dieter Lüst

Rasmus Mackeprang

Dennis Mackin

Nobuhiro Maekawa

Carsten Magass

K.T. Mahanthappa

Rakhi Mahbubani

Prolay Mal

Giovanni Marchiori

Danny Marfatia

Nancy Marinelli

Christopher Marino

Anja Marold

Stephen Martin

Antonio Masiero

John Mason

Anupam Mazumdar

Anibal Medina

Bernhard Meirose

Isabell-A. Melzer-

Pellmann
Centre for Research in String Theory, QMUL

Max-Planck-Institut

UCSB

LPSC Grenoble

Michigan State University

University of Oregon

Princeton University

University of Hawaii

University of California

LANL

UIC

The University of Arizona

Brown University

Institute for Advanced Study

DESY Zeuthen

Johns Hopkins University

University of Oxford

Università di Milano

University of Birmingham

LPSC

UC Riverside

Max Planck Institute

UC Riverside

National Central University

University of Wisconsin, Madison

Universidad Autonoma Metropolitana

Iztapalapa

Stanford University

University of Chicago

Stony Brook University

Dept. of Physics, Un. Patras

U. C. Riverside \& SLAC

Princeton University

California Institute of Technology/Columbia

University

IRFU,CEA-Saclay

Argonne/Northwestern

LMU-Muenchen

CERN

Rice University/Dzero

Nagoya University

RWTH Aachen University

University of Colorado at Boulder

Fermilab

The University of Arizona

LPNHE, Paris

University of Kansas

Univ. of Notre Dame

Indiana University

PI, Uni Bonn

Northern Illinois University

Univ. of Padova and INFN, Padova

Harvard University

Lancaster

UC Davis

Freiburg University

DESY 


\begin{tabular}{|c|c|}
\hline Arjun Menon & University of Michigan \\
\hline Paolo Meridiani & CERN \\
\hline Luca Merlo & University of Padua \& INFN Padua \\
\hline Philippe Mermod & Stockholm University \\
\hline Arnd Meyer & RWTH Aachen \\
\hline Octavian Micu & TU Dortmund \\
\hline David Miller & University of Glasgow \\
\hline David Milstead & Stockholm University \\
\hline Yukihiro Mimura & University of Maryland \\
\hline Shulamit Moed & Harvard University \\
\hline Rabindra Mohapatra & University of Maryland \\
\hline Myriam Mondragon & Inst. de Fisica, UNAM \\
\hline David Morrissey & Harvard University \\
\hline Moshe Moshe & Technion - Israel Institute of Technology \\
\hline $\begin{array}{l}\text { Biswarup } \\
\text { Mukhopadhyaya }\end{array}$ & Harish-Chandra Research Institute \\
\hline Shoaib Munir & $\begin{array}{l}\text { Insituto de Fisica, Universidad Nacional } \\
\text { Autonoma de Mexico }\end{array}$ \\
\hline Carlos Munoz & Universidad Autonoma de Madrid \& IFT \\
\hline William Murray & RAL \\
\hline Azar Mustafayev & University of Kansas \\
\hline Keiko Nagao & Nagoya University \\
\hline Eita Nakamura & University of Tokyo \\
\hline Satyanarayan Nandi & Oklahoma State University \\
\hline Pran Nath & Northeastern University \\
\hline Brent Nelson & Northeastern University \\
\hline Roman Nevzorov & University of Glasgow \\
\hline Kaixuan $\mathrm{Ni}$ & Shanghai Jiao Tong University \\
\hline Hans Peter Nilles & Univ. Bonn \\
\hline Carlos Nunez & Swansea University \\
\hline Ben O'Leary & RWTH Aachen University \\
\hline Takemichi Okui & $\begin{array}{l}\text { Johns Hopkins Univ \& Univ of Maryland, } \\
\text { College Park }\end{array}$ \\
\hline Ali Ovgun & Izmir Institute of Technology \\
\hline Nurcan Ozturk & University of Texas at Arlington \\
\hline Katarina Pajchel & University of Oslo \\
\hline Sean Paling & Sheffield University \\
\hline Sophy Palmer & $\begin{array}{l}\text { Institute for Particle Physics } \\
\text { Phenomenology, Durham University }\end{array}$ \\
\hline Grigoris Panotopoulos & LMU Munich \\
\hline Seong Chan Park & IPMU, University of Tokyo \\
\hline Thomas Paul & Northeastern University \\
\hline Gil Paz & Institute for Advanced Study, Princeton \\
\hline Maxim Perelstein & Cornell University \\
\hline Kala Perkins & UCSB \\
\hline Piergiorgio Picozza & INFN and University of Rome Tor Vergata \\
\hline Aaron Pierce & University of Michigan \\
\hline Maurizio Pierini & CERN \\
\hline Apostolos Pilaftsis & University of Manchester \\
\hline David Poland & Harvard University \\
\hline Giacomo Polesello & INFN Sezione di Pavia \\
\hline Eduardo Pontón & Columbia University \\
\hline Werner Porod & Uni. Wurzburg \\
\hline Xavier Portell & Albert-Ludwigs-Universitaet Freiburg \\
\hline Stefano Profumo & UC Santa Cruz \\
\hline Ivica Puljak & University of Split (FESB) \\
\hline Stuart Raby & The Ohio State University \\
\hline Arvind Rajaraman & University of California, Irvine \\
\hline Surjeet Rajendran & SLAC \\
\hline Lisa Randall & Harvard \\
\hline
\end{tabular}


Kumar Rao

Salvatore Rappoccio

Fedor Ratnikov

Ronald Remington

Leonidas Resvanis

Juergen Reuter

Marco Ricci

Adam Ritz

Andrea Rizzi

Thomas Rizzo

Krzysztof Rolbiecki

Probir Roy

Tuhin Roy

Alberto Ruiz-Jimeno

Timo Ruppell

Asli Sabanci

Yutaka Sakamura

Miguel-Angel Sanchis-

Lozano

Christian Sander

Pearl Sandick

Jose Santiago Perez

Yudi Santoso

Veronica Sanz

Gregory Schott

Nicolas Schul

Philip Schuster

Pedro Schwaller

Goran Senjanovic

Jihye Seo

Nicholas Setzer

Nausheen Shah

Chung-Lin Shan

Jing Shao

Gabe Shaughnessy

Jessie Shelton

Marc Sher

Mikhail Shifman

Satoshi Shirai

Yuri Shirman

Jing Shu

Vladislav Simak

Pekka Sinervo

Kuver Sinha

Therese Sjursen

Scott Snyder

Roberta Sparvoli

Christian Spethmann

Sogee Spinner

Martin Spinrath

Paola Squillacioti

Oscar Stål

Markus Stoye

David Straub

Raimund Stroehmer

John Strologas

Derek Strom

Sergey Sukhoruchkin
Helsinki Institute of Physics

Johns Hopkins University

KIT, Karlsruhe

University of Florida

NESTOR Institute

University of Freiburg

Istituto Nazionale di Fisica Nucleare

University of Victoria

ETH Zurich

SLAC

IPPP Durham

Saha Institute

University of Oregon

Instituto de Fisica de Cantabria (IFCA, CSIC-Univ. Cantabria)

HIP

University of Helsinki

RIKEN

IFIC - Valencia University

University of Hamburg

UT Austin

ETH Zurich

IPPP, University of Durham

Boston University

University of Karlsruhe

UCLouvain

SLAC

University of Zurich

ICTP

Harvard University

University of Melbourne

University of Chicago

Seoul National University

$\mathrm{U}$ of Michigan

Northwestern University / Argonne

National Laboratory

Rutgers University

William and Mary

FTPI, Univ. of Minnesota

University of Tokyo

UC Irvine

IPMU

Czech Technical University, Prague

University of Toronto

Texas A\&M University

University of Bergen

BNL

UNiversity of Rome Tor Vergata and INFN

Cornell University

University of Wisconsin, Madison

Max-Planck-Institut

INFN Pisa \& Siena University

Uppsala University

Imperial College London

TU München

LMU Munich

University of New Mexico

Northwestern University

Petersburg Nuclear Physics Institute 